Discrete Comput Geom 36:167-204 (2006)

DOI: $10.1007 / \mathrm{s} 00454-005-1214-\mathrm{y}$

\title{
Sphere Packings, V. Pentahedral Prisms
}

\author{
Samuel P. Ferguson \\ 5960 Millrace Court B-303, \\ Columbia, MD 21045, USA \\ samf2@ comcast.net
}

\begin{abstract}
This paper is the fifth in a series of papers devoted to the proof of the Kepler conjecture, which asserts that no packing of congruent balls in three dimensions has density greater than the face-centered cubic packing.

In this paper we prove that decomposition stars associated with the plane graph of arrangements we term pentahedral prisms do not contravene. Recall that a contravening decomposition star is a potential counterexample to the Kepler conjecture. We use interval arithmetic methods to prove particular linear relations on components of any such contravening decomposition star. These relations are then combined to prove that no such contravening stars exist.
\end{abstract}

\section{Introduction}

Pentahedral prisms come remarkably close to achieving the optimal score of $8 \mathrm{pt}$, that achieved by the decomposition stars of the face-centered cubic lattice packing. In this sense we consider pentahedral prisms to be "worst-case" decomposition stars.

Pentahedral prisms constituted a counterexample to an early version of Hales's approach to a proof of the Kepler conjecture, and have always been a somewhat thorny obstacle to the proof of the conjecture. Relations required to treat pentahedral prisms are delicate in contrast to the more general bounds which suffice to treat other decomposition stars.

\section{Pentahedral Prisms}

Recall that a contravening decomposition star is a potential counterexample to the Kepler conjecture. The subject of this paper is a particular class of potentially contravening decomposition stars. 
We use the term pentahedral prisms to refer to this class of potentially contravening decomposition stars, and refer to a decomposition star in this class as a pentahedral prism. This class is defined by the plane graph in Fig. 15.1.

An example of an arrangement with such a graph is depicted in Fig. 15.2.

A pentahedral prism is characterized by the arrangement and combinatorics of its standard regions. It is composed of ten triangular standard regions, and five quadrilateral standard regions.

The ten triangles are arranged in two pentahedral caps, five triangles arranged around a common vertex. The five quadrilaterals lie in a band between the two caps. See Fig. 15.3.

Recall that the standard cluster attached to a triangular standard region is a quasiregular tetrahedron. Likewise, the standard cluster attached to a quadrilateral is a quad cluster. We use the term pentahedral cap to refer to both the standard regions and the quasi-regular tetrahedrons which comprise it.

\subsection{The Main Theorem}

We begin by recalling various definitions from the second paper, Formulation [HF]. The constant $p t$ is introduced in Definition 3.6. Similarly, score is defined in Theorem 3.5, as well as Definition 7.8 and Remark 7.20. We denote the score of a region $R$ by $\sigma(R)$.

Theorem 15.1. Each pentahedral prism $P$ satisfies

$$
\sigma(P) \leq\left(8-\varepsilon_{0}\right) p t
$$

for $\varepsilon_{0}=10^{-8}$. Hence there are no contravening pentahedral prisms.

The next section introduces a series of propositions which prove the main theorem. The first proposition restricts our attention to a set of potentially contravening pentahedral prisms. Subsequent propositions provide a collection of relations which we use to prove the main theorem.

\subsection{Propositions}

The function sol(.) is introduced in Definition 7.5. The function $\operatorname{dih}(\cdot)$ is introduced in Definition 4.12.

We present computations using auxiliary bounds which imply the main result of the paper, that the score of any pentahedral prism is strictly less than $8 \mathrm{pt}$.

Recall from Section 7.4 that the score decomposition for a decomposition star $S$ takes the form

$$
\sigma(S)=\sum_{R} \sigma(R)
$$

where $R$ runs over the standard clusters in $S$. 


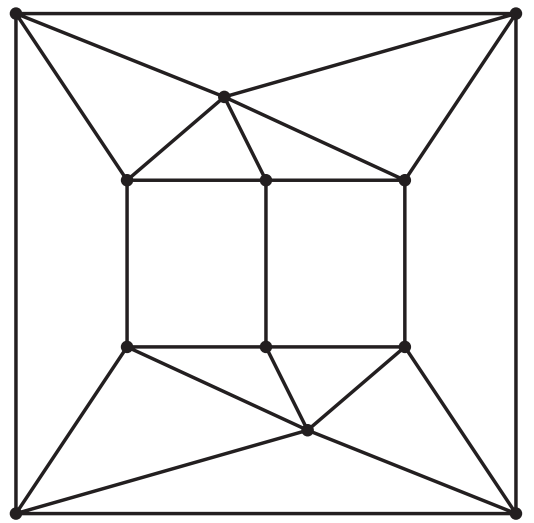

Fig. 15.1. The plane graph of a pentahedral prism.

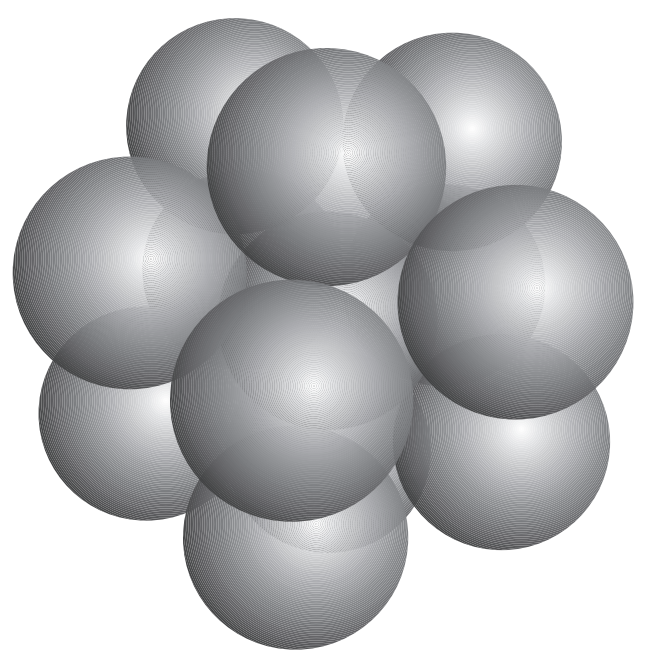

Fig. 15.2. Spheres in a pentahedral prism arrangement.

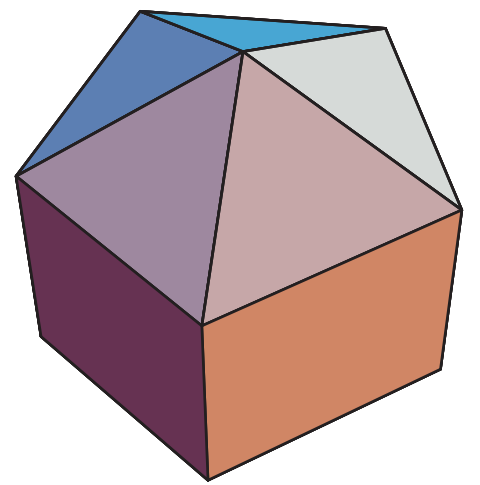

Fig. 15.3. The faces of a pentahedral prism. 
In the case of a pentahedral prism $P$, the score $\sigma(P)$ decomposes as

$$
\sigma(P)=\sum_{i=1}^{10} \sigma\left(T_{i}\right)+\sum_{j=1}^{5} \sigma\left(Q_{j}\right)
$$

with the triangular regions $T_{i}$ numbered so that the two pentahedral caps $C_{i}$ consist of $\left\{T_{i}: 1 \leq i \leq 5\right\},\left\{T_{i}: 6 \leq i \leq 10\right\}$ and $Q_{j}$ denotes the quad clusters. Thus

$$
\sigma\left(C_{1}\right)=\sum_{i=1}^{5} \sigma\left(T_{i}\right), \quad \sigma\left(C_{2}\right)=\sum_{i=6}^{10} \sigma\left(T_{i}\right) .
$$

The following proposition gives basic inequalities which we use to form a restricted set of pentahedral prisms.

Proposition 15.2. A pentahedral prism $P$ satisfies the bound

$$
\sigma(P) \leq\left(8-\varepsilon_{0}\right) p t
$$

for $\varepsilon_{0}=10^{-8}$ provided that any one of the following conditions holds:

1. P contains a tetrahedron $T$ such that

$$
\sigma(T) \leq-0.52 p t
$$

2. P contains a quad cluster $Q$ such that

$$
\sigma(Q) \leq-1.04 p t
$$

3. P contains a pentahedral cap $C$ such that

$$
\sigma(C) \leq 3.48 p t
$$

Proof. We use the following scoring bounds proved earlier for any admissible decomposition star.

First, Lemma 8.10 states that any quasi-regular tetrahedron $T$ satisfies

$$
\sigma(T) \leq 1 p t
$$

Theorem 8.4 states that any quad cluster $Q$ satisfies

$$
\sigma(Q) \leq 0
$$

Next, a pentahedral cap $C$ consists of five quasi-regular tetrahedra $T_{i}$ sharing a common distinguished edge. At one end of the distinguished edge is the distinguished vertex $v=0$ which is the center of the decomposition star $P$. Each $T_{i}$ has context $c_{i}=(5,0)$. 
Lemmas 10.6 (with $k=1$ and $r=5$ ) and 10.7 state that any pentahedral cap $C_{i}$ satisfies

$$
\sigma\left(C_{i}\right)=\sum_{i=1}^{5} \sigma\left(T_{i}, c_{i}, v\right) \leq\left(4.52-\varepsilon_{0}\right) p t
$$

with $\varepsilon_{0}=10^{-8}$.

1. Suppose that some $\sigma(T) \leq-0.52 \mathrm{pt}$, with $T$ contained in a pentahedral cap $C_{1}$. Then the inequalities above give

$$
\begin{aligned}
\sigma & \leq-0.52 p t+4(1 p t)+\sigma\left(C_{2}\right)+\sum_{j=1}^{5} \sigma\left(Q_{j}\right) \\
& \leq 3.48 p t+\left(4.52-\varepsilon_{0}\right) p t+5(0)=\left(8-\varepsilon_{0}\right) p t .
\end{aligned}
$$

2. Suppose that some quad cluster $\sigma\left(Q_{j}\right) \leq-1.04 p t$. Then

$$
\begin{aligned}
\sigma(P) & \leq \sigma\left(C_{1}\right)+\sigma\left(C_{2}\right)+(-1.04 p t)+4(0) \\
& \leq 2\left(\left(4.52-\varepsilon_{0}\right) p t\right)-(1.04 p t)=\left(8-2 \varepsilon_{0}\right) p t .
\end{aligned}
$$

3. Suppose that some pentahedral cap $C_{1}$ has $\sigma\left(C_{1}\right) \leq 3.48 p t$. Then the inequalities above give

$$
\sigma(P) \leq(3.48 p t)+\left(4.52-\varepsilon_{0}\right) p t+5(0)=\left(8-\varepsilon_{0}\right) p t .
$$

This completes the proof.

Definition 15.3. A PC pentahedral prism is a pentahedral prism such that:

1. All tetrahedra $T$ have $\sigma(T) \geq-0.52 p t$.

2. All quad clusters have $\sigma(Q) \geq 1.04 p t$.

3. All pentahedral caps have $\sigma(C) \geq 3.48 p t$.

The configuration arises as a pointwise limit of configurations in which (1)-(3) hold with strict inequality. A strict PC pentahedral prism is one in which (1)-(3) each hold with strict inequality.

All remaining propositions apply to $\mathrm{PC}$ pentahedral prisms. This restriction improves the quality of the bounds which we are able to prove on components of a pentahedral prism.

The following two propositions contain linear relations which imply the main theorem. We defer their proofs to the next section.

Proposition 15.4. For a quasi-regular tetrahedron $T$ in a $P C$ pentahedral prism, the following linear inequality holds between $\sigma(T)$, the spherical angle $\operatorname{sol}(T)$ (at the central vertex common to the five tetrahedra in the pentahedral cap), and the dihedral angle 
$\operatorname{dih}(T)$ associated with the first edge of the tetrahedron (that is, the edge common to the five tetrahedra in a pentahedral cap):

$$
\sigma(T)+m \operatorname{sol}(T)+a\left(\operatorname{dih}(T)-\frac{2 \pi}{5}\right)-b_{c} \leq 0 .
$$

Here $a=0.0739626, b_{c}=0.253095$, and $m=0.3621$.

Proposition 15.5. For a quad cluster $Q$ in a PC pentahedral prism, the following linear inequality holds between $\sigma(Q)$ and the spherical angle $\operatorname{sol}(Q)$ :

$$
\sigma(Q)+m \operatorname{sol}(Q)-b_{q} \leq 0 .
$$

Here $b_{q}=0.49246$ and again $m=0.3621$.

From Propositions 15.4 and 15.5 we can deduce the following theorem.

Theorem 15.6. Each PC pentahedral prism P satisfies the score bound

$$
\sigma(P) \leq 7.9997 p t .
$$

Proof. Propositions 15.4 and 15.5 provide linear relations on all of the standard clusters in a PC pentahedral prism $P$. We combine these relations to prove the required score bound.

Invoking Proposition 15.4 for the five quasi-regular tetrahedrons $\left\{T_{i}: i=1 \ldots 5\right\}$ from a pentahedral cap, we find

$$
\sum_{i=1}^{5} \sigma\left(T_{i}\right)+m \sum_{i=1}^{5} \operatorname{sol}\left(T_{i}\right)+a \sum_{i=1}^{5}\left(\operatorname{dih}\left(T_{i}\right)-\frac{2 \pi}{5}\right)-5 b_{c} \leq 0 .
$$

Summing over both pentahedral caps and using the relation that the sum of the five dihedral angles in a pentahedral cap is $2 \pi$,

$$
\sum_{i=1}^{5} \operatorname{dih}\left(T_{i}\right)=2 \pi
$$

we find

$$
\sum_{i=1}^{10} \sigma\left(T_{i}\right)+m \sum_{i=1}^{10} \operatorname{sol}\left(T_{i}\right)-10 b_{c} \leq 0 .
$$

We represent the tetrahedra from the second pentahedral cap by the indices $i=6 \ldots 10$.

Invoking Proposition 15.5 for the five quad clusters $\left\{Q_{i}: i=11 \ldots 15\right\}$, and using the fact that the sum of the solid angles is $4 \pi$,

$$
\sum_{i=1}^{10} \operatorname{sol}\left(Q_{i}\right)+\sum_{j=11}^{15} \operatorname{sol}\left(Q_{j}\right)=4 \pi,
$$


we find

$$
\sum_{i=1}^{10} \sigma\left(T_{i}\right)+\sum_{j=11}^{15} \sigma\left(Q_{j}\right)+4 \pi m-5 b-10 b_{c} \leq 0 .
$$

Therefore,

$$
\sigma(P) \leq 5 b+10 b_{c}-4 \pi m .
$$

Substituting the values of $b, b_{c}, m$, and $p t$, we find that the score of a PC pentahedral prism is less than $7.9997 \mathrm{pt}$.

Assuming Proposition 15.2 and Theorem 15.6 we can prove Theorem 15.1.

Proof of Theorem 15.1. Given a pentahedral prism $P$, it is either PC or it is not. In the former case its score is bounded by $7.9997 \mathrm{pt}$. In the latter case its score is bounded by $\left(8-10^{-8}\right) p t$. In both cases its score is bounded by $\left(8-10^{-8}\right) p t$.

Remark 15.7. The score bound in Theorem 15.1 is weaker than what is possible to prove. In the interest of simplifying the exposition as well as the required computations, we establish this weaker bound which suffices for this part of the proof of the Kepler conjecture.

\section{The Main Propositions}

In the first section we recall the definition of score, and introduce some local notation. In the next section we recall the notion of dimension reduction, and prove its validity for some relevant cases. In the following section we prove Proposition 15.4. In the remaining sections we prove Proposition 15.5.

\subsection{Scoring}

The development of a scoring function is central to the proof of the Kepler conjecture. Its definition is therefore somewhat complicated. Fortunately, in our treatment of the pentahedral prism we are able to restrict our attention to only a few cases in the scoring system.

Recall that score is defined in Theorem 3.5, as well as Definitions 7.6 and 7.8 and Remark 7.20. See Remark 7.23 for a simplified version of the scoring function for quarters.

In our context, the score $\sigma(\cdot)$ breaks into four different scoring types: gma( $(\cdot), \operatorname{vor}(\cdot)$, octavor( $(\cdot)$, and Voronoi.

$\operatorname{gma}(\cdot)$ applies to quasi-regular tetrahedrons and quarters, and is introduced as $\Gamma(\cdot)$ in Definition 7.6. We frequently use the term compression as an alias for $g m a(\cdot)$. This alias was introduced in Section 7.6. 
$\operatorname{vor}(\cdot)$ is the score determined by the analytic continuation of the Voronoi volume associated with the distinguished vertex of a tetrahedron, and corresponds to s-vor(.) in Definition 7.6.

We let octavor( $(\cdot)$ denote the score of an upright quarter in context $(4,0)$ which is not scored by compression. In this case, octavor(.) is the average of two vor(.) scores.

Voronoi scoring, which we also refer to as pure Voronoi scoring, is $\operatorname{vor}_{R}(D)$ from Remark 7.20.

\subsection{Dimension Reduction}

The relations on tetrahedra required for the scoring bound on decomposition stars are typically six-dimensional, as they are formulated in terms of the edge lengths of a tetrahedron. For a quad cluster, they can be even higher-dimensional. For high-dimensional relations, the method of subdivision becomes very expensive, computationally speaking.

We define a simplification which reduces the dimension of the required computations. This simplification therefore reduces the computational expense of the verification of a relation.

We refer to this simplification as dimension-reduction. We apply this simplification for three different scoring types: compression, vor analytic, and Voronoi. These scoring functions are introduced in Definitions 7.6 and 7.8. See Remark 7.23 for a simplified version of the scoring function for quarters.

Theorem 16.1 (Dimension-Reduction). Given a tetrahedron $T$ with a fixed scoring type (one of compression, vor analytic, or Voronoi), the deformation consisting of moving a vertex $v_{i}$ along the edge $\left(0, v_{i}\right)$ towards the origin increases the score of the tetrahedron.

Note that this deformation holds the solid angle at the origin fixed. See Fig. 16.1. Since the reduction may be performed until either a scoring system or an edge-length constraint is met, this argument reduces the number of free parameters for the verification, thus reducing the dimension and complexity of the verification of a relation.

Proof. There are three cases to consider: compression scoring, vor analytic scoring and Voronoi scoring. This technique was introduced in Proposition 8.7.1 of [Ha6] for compression-scoring, and is proved there in the compression case.

Next we consider vor analytic-scored tetrahedra. The validity of the same reduction for vor analytic-scored tetrahedra is obvious if the tip of the Voronoi cell does not protrude. If the tip does protrude, we must use the analytic continuation for the Voronoi volume. In this case the validity of the reduction is not obvious.

The geometric constraint of moving a vertex along an edge can easily be stated analytically in terms of the original edge lengths, $\left(y_{1}, y_{2}, y_{3}, y_{4}, y_{5}, y_{6}\right)$. This action depends on a single parameter, the distance of the vertex $v_{1}$ from the origin, which we 


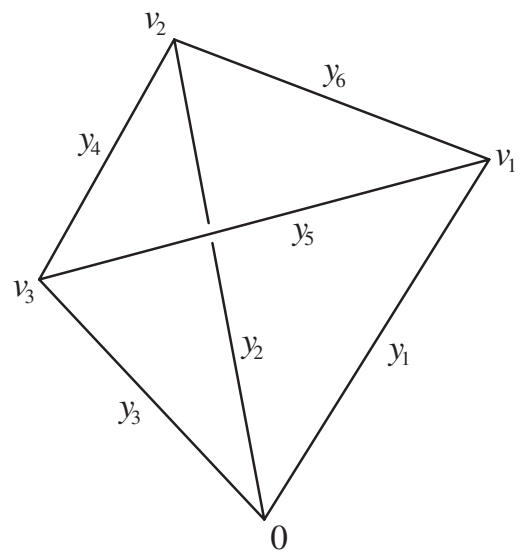

Fig. 16.1. Tetrahedron with distinguished vertex and labeled edges.

call $t$. The new edge lengths are given by

$$
\left(t, y_{2}, y_{3}, y_{4}, \sqrt{t^{2}+y_{3}^{2}-\frac{t\left(y_{1}^{2}+y_{3}^{2}-y_{5}^{2}\right)}{y_{1}}}, \sqrt{t^{2}+y_{2}^{2}-\frac{t\left(y_{1}^{2}+y_{2}^{2}-y_{6}^{2}\right)}{y_{1}}}\right) .
$$

Recall from Section 8.6.3 of [Ha6] that the formula for the analytic Voronoi volume is a rational function of $\chi, u, \sqrt{\Delta}$, and $x_{i}$, where $x_{i}=y_{i}^{2}$. Further recall that $\chi, u$, and $\Delta$ are all polynomial functions in $x_{i}$ that are defined in Sections 8.1 and 8.2 of [Ha6].

Substituting the computed edge lengths in the formula for the analytic Voronoi volume, taking the partial derivative with respect to $t$, replacing $t$ with $y_{1}$, multiplying by the positive term

$$
8 \sqrt{\Delta} u\left(x_{1}, x_{3}, x_{5}\right) u\left(x_{1}, x_{2}, x_{6}\right) / y_{1},
$$

and then simplifying, we end up with a large homogeneous polynomial in $x_{i}$ of degree 6 , which is too ugly to exhibit here (having ninety-one terms).

Evaluating this polynomial over all possible quasi-regular tetrahedrons and quarters, we find that it is positive.

Therefore the volume is increasing in $t$, so to increase the score we should push the vertex in along the edge. The verification of the sign of the polynomial is found in Calculation 17.4.5.1. This completes the case of vor analytic-scored tetrahedra.

The final case is Voronoi scoring. The deformation does not change the solid angle of the tetrahedron. The only term of the Voronoi score that changes is a negative constant times a volume. The contraction of the tetrahedron decreases this volume, and increases the score. The validity of a similar reduction argument for Voronoi scoring of a tetrahedron is now obvious. 


\subsection{Proof of Proposition 15.4}

It suffices to prove Proposition 15.4 for strict PC pentahedral prisms. Each nonstrict PC pentahedral prism is a pointwise limit of strict ones of the same combinatorial type, so the inequality in the conclusion of the proposition will hold for nonstrict PC pentahedral prisms by continuity.

We use three separate computations to construct and prove Proposition 15.4. First, we prove a relation between dihedral angle and score. We then show that if the dihedral angle of a tetrahedron in a pentahedral cap exceeds a certain bound, then the associated pentahedral prism is not a strict PC pentahedral prism. We call such a bound a dihedral cutoff. This cutoff allows us to prove the final bound.

In the following discussion, $\operatorname{dih}(T)$ refers to the dihedral angle associated with the first edge of a quasi-regular tetrahedron $T, \sigma(T)$ refers to the compression score of the tetrahedron, and $\operatorname{sol}(T)$ refers to the solid angle at the distinguished vertex. We restrict our attention to quasi-regular tetrahedrons whose score exceeds $-0.52 \mathrm{pt}$, as otherwise the associated pentahedral prism cannot contravene.

The first relation is

$$
\sigma(T) \leq a_{1} \operatorname{dih}(T)-a_{2},
$$

where $a_{1}=0.3860658808124052$ and $a_{2}=0.4198577862$. Calculation 17.4.1.1 provides the verification of this relation.

Lemma 16.2. If a pentahedral prism has a pentahedral cap that contains a quasiregular tetrahedron $T$ with dihedral angle $\operatorname{dih}(T) \geq d_{0}$, where $d_{0}=1.4674$, then it is not a strict PC pentahedral prism.

Proof. Applying relation (16.1) to four quasi-regular tetrahedrons $T_{i}$ forming part of a strict PC pentahedral prism, we find

$$
\sum_{i=1}^{4} \sigma\left(T_{i}\right) \leq a_{1} \sum_{i=1}^{4} \operatorname{dih}\left(T_{i}\right)-4 a_{2} .
$$

Applying the relation

$$
\operatorname{dih}\left(T_{5}\right)=2 \pi-\sum_{i=1}^{4} \operatorname{dih}\left(T_{i}\right)
$$

and adding $\sigma\left(T_{5}\right)$ to both sides of relation (16.2), we find

$$
\sum_{i=1}^{5} \sigma\left(T_{i}\right) \leq \sigma\left(T_{5}\right)+a_{1}\left(2 \pi-\operatorname{dih}\left(T_{5}\right)\right)-4 a_{2} .
$$

The left-hand side represents the score of the pentahedral cap. If the right-hand side does not exceed $3.48 \mathrm{pt}$, then the pentahedral prism is not a strict PC pentahedral prism.

We assert that if $\operatorname{dih}(T) \geq d_{0}$, the right-hand side

$$
\sigma\left(T_{5}\right)+a_{1}\left(2 \pi-\operatorname{dih}\left(T_{5}\right)\right)-4 a_{2}
$$


does not exceed $3.48 \mathrm{pt}$. Equivalently, we prove that $\operatorname{dih}(T) \geq d_{0}$ implies

$$
\sigma(T)-a_{1} \operatorname{dih}(T) \leq 3.48 p t-2 \pi a_{1}+4 a_{2},
$$

which is verified in Calculation 17.4.1.2.

We conclude that if $\operatorname{dih}(T) \geq d_{0}$ then the pentahedral prism cannot be a strict PC pentahedral prism.

Hence we may restrict our attention to quasi-regular tetrahedrons whose dihedral angle does not exceed the dihedral cutoff $d_{0}$.

Using the dihedral cutoff, we establish the final relation,

$$
\sigma(T)+m \operatorname{sol}(T)+a(\operatorname{dih}(T)-2 \pi / 5)-b_{c} \leq 0
$$

via Calculation 17.4.1.3. This completes the proof of Proposition 15.4.

\subsection{Proof of Proposition 15.5: Top Level}

It suffices to prove Proposition 15.5 for strict PC pentahedral prisms, by a similar argument to that used for Proposition 15.4.

Recall from Definition 7.15 that a quad cluster is a standard region that is a quadrilateral. Quad clusters can be classified as follows:

1. Flat quad clusters.

2. Octahedra.

3. Pure Voronoi quad clusters.

4. Mixed quad clusters.

We subdivide the third class into acute and obtuse types. See Section 10.4 for a discussion on the classification of quad clusters. By Lemma 10.14, the score of a mixed quad cluster is less than -1.04 pt. A PC pentahedral prism therefore cannot contain a mixed quad cluster, so the bound of Proposition 15.5 holds trivially for this class.

We treat the remaining classes in the following sections.

\subsection{Proof of Proposition 15.5: Flat Quad Clusters}

Recall that a flat quarter is a quarter whose long edge is opposite its distinguished vertex.

Lemma 16.3. Given a flat quarter $Q$ with $\sigma(Q) \geq-1.04$ pt, the relation

$$
\sigma(Q) \leq-m \operatorname{sol}(Q)+b / 2
$$

holds.

Proof. Label the diagonal of a flat quarter $y_{6}$.

Flat quarters may be scored using either compression or vor scoring. We treat each case separately. 
First, suppose that we wish to prove the bound for compression scored quarters. This means that the circumradii of the two faces adjacent to the long diagonal do not exceed $\sqrt{2}$. We subdivide the verification into Calculation 17.4.2.1, a computation where we apply dimension-reduction and partial derivative information, and Calculation 17.4.2.2, a boundary verification, where we restrict our attention to cells which lie on the boundary between compression and vor scoring.

Second, we treat the vor-scoring case. In this case we prove the bound for vor-scored quarters. This means that at least one of the circumradii of the two faces adjacent to the long diagonal is at least $\sqrt{2}$. This verification is somewhat more complex than the compression case. We subdivide the verification into

1. Verification that the first three partials are negative on a small cell containing the corner (Calculation 17.4.2.3).

2. Verification of the bound on that small cell containing the corner, using the property that the first three partials are negative (Calculation 17.4.2.4).

3. A computation where we apply dimension-reduction and partial derivative reduction, omitting the corner cell (Calculation 17.4.2.5).

4. A boundary verification, where we restrict our attention to cells which lie on the boundary between compression and vor scoring, again omitting the corner cell (Calculation 17.4.2.6).

These calculations complete the proof of Lemma 16.3.

We are now prepared to prove Proposition 15.5 for flat quad clusters.

Flat quad clusters are composed of two flat quarters, whose common face includes the long edge.

By Proposition 15.2, we restrict our attention to flat quarters whose score exceeds $-1.04 \mathrm{pt}$, recalling the fact that the score of flat quarters is nonpositive.

Invoking Lemma 16.3 for each flat quarter and adding the relations, we arrive at the desired bound for flat quad clusters. This completes the proof.

\subsection{Proof of Proposition 15.5: Octahedra}

Recall that quartered octahedra, a type of quad cluster, are composed of four upright quarters arrayed around their common long edge (called the diagonal) so that each face containing the common edge is shared by two quarters.

We are required to prove a relation of the form

$$
\sigma(H)+m \operatorname{sol}(H)-b \leq 0,
$$

where $\sigma(H)$ denotes the score of an octahedron $H, \operatorname{sol}(H)$ denotes the solid angle associated with the distinguished vertex, and $m$ and $b$ are positive constants. By Proposition 15.2 , we restrict our attention to octahedra whose score exceeds $-1.04 \mathrm{pt}$.

Our treatment of octahedra, as usual, is comprised of a number of auxiliary computations. We prove bounds on upright quarters which are part of an octahedron, and then combine these bounds to deduce the required bound on octahedra in general. 
The scoring function $\sigma(\cdot)$ for upright quarters is either compression (denoted by gma $(\cdot))$ or an average of two vor(.) scores, which we continue to refer to as vor-scoring. See Remark 7.23 for a simplified version of the scoring rules.

Due to the complex nature of octahedra, we consider a number of subcases. These cases are partitioned according to the length of the diagonal and the scoring system applied to the upright quarters.

Using a dihedral summation argument, we eliminate octahedra whose diagonal lies in the range [2.51, 2.716].

Next, we treat the case where the diagonal lies in the range $[2.716,2 \sqrt{2}]$. Using a dihedral correction term, we prove the bound for octahedra which are completely compression-scored, and octahedra which are completely vor-scored.

The remaining cases consist of octahedra which contain either two or three vor-scored quarters. (Since a quarter is vor-scored if one of the faces containing the diagonal has circumradius $\sqrt{2}$ or greater, it is not possible for an octahedron to contain only one vor-scored quarter.) We treat these cases using an additional correction term.

In all computations involving octahedra, we label the diagonal $y_{1}$.

In order to simplify the computations, we first prove an auxiliary cutoff bound. This first bound reduces the size of the cell over which we must conduct our search, as per Proposition 15.2.

Lemma 16.4. If an upright quarter contains an edge numbered 2, 3, 5, or 6 whose length is not less than 2.2, then its score is less than or equal to $-0.52 \mathrm{pt}$.

Proof. This is Calculation 17.4.3.1.

Since such an edge is shared by another upright quarter in the same octahedron, the score of the associated octahedron must fall below $-1.04 \mathrm{pt}$.

We restrict our search accordingly.

Lemma 16.5. The score of an octahedron $H$ with upright diagonal in the range $[2.51,2.716]$ is less than or equal to $-1.04 \mathrm{pt}$.

Proof. In Calculation 17.4.3.2 we prove a bound of the form

$$
\sigma(S)+c \operatorname{dih}(S) \leq d
$$

on upright quarters $S$, where $c=0.1533667634670977$ and $d=0.2265$. Adding the bound for four quarters $S_{i}$ forming an octahedron, we find

$$
\sum_{i=1}^{4} \sigma\left(S_{i}\right)+c \sum_{i=1}^{4} \operatorname{dih}\left(S_{i}\right) \leq 4 d .
$$

Using the fact that the sum of the dihedral angles is $2 \pi$, we find that

$$
\sigma(H) \leq-2 \pi c+4 d
$$

for such an octahedron $H$. 
A computation involving the constants $c$ and $d$ shows that the score is less than $-1.04 p t$.

Again invoking Proposition 15.2, we need only consider octahedra whose diagonal lies in the range $[2.716,2 \sqrt{2}]$.

Using this assumption, we prove bounds of the form

$$
\sigma(S)+m \operatorname{sol}(S)+\alpha \operatorname{dih}(S) \leq \frac{b}{4}+\alpha \frac{\pi}{2}
$$

and

$$
\sigma(S)+m \operatorname{sol}(S)+\alpha \operatorname{dih}(S)+\beta x_{1} \leq \frac{b}{4}+\alpha \frac{\pi}{2}+8 \beta,
$$

where $\operatorname{dih}(S)$ refers to the dihedral angle associated with the diagonal, $\sigma(S)$ refers to the scoring scheme appropriate for a particular upright quarter $S$, and $x_{1}$ refers to the square of the length of the diagonal. We choose $\alpha$ and $\beta$ according to the scoring scheme.

Appropriate values for the correction terms involving $\alpha$ and $\beta$ were determined by experimentation.

Choosing $\alpha=0.14$, we prove (16.6) for compression-scored quarters with diagonal in the interval $[2.716,2 \sqrt{2}$ ] (Calculation 17.4.3.3). Using the same $\alpha$, we prove (16.6) for vor-scored quarters with diagonal in the range [2.716, 2.81] (Calculation 17.4.3.4).

Choosing $\alpha=0.054, \beta=0.00455$, we prove (16.7) for compression-scored quarters with diagonal in $[2.81,2 \sqrt{2}]$ (Calculation 17.4.3.5). Choosing the same $\alpha$, but $\beta=-0.00455$, we prove (16.7) for vor-scored quarters with diagonal in $[2.81,2 \sqrt{2}]$ (Calculation 17.4.3.6).

Note that for vor-scored quarters, the first inequality is a relaxation of the second, since $\beta$ is negative.

The verification of each of these inequalities involves a computation where we apply dimension-reduction and partial derivative information, and a boundary verification, where we restrict our attention to cells which lie on the boundary between compression and vor analytic scoring. Note that the dimension-reduction step for relation (16.7) is complicated by the presence of the $\beta x_{1}$ term.

Lemma 16.6. Proposition 15.5 holds for octahedra with upright diagonals in the range $[2.716,2 \sqrt{2}]$.

Proof. Summing inequality (16.6) over the four quarters $S_{i}$ of an octahedron, we find

$$
\sum_{i=1}^{4} \sigma\left(S_{i}\right)+m \sum_{i=1}^{4} \operatorname{sol}\left(S_{i}\right)+\alpha \sum_{i=1}^{4} \operatorname{dih}\left(S_{i}\right) \leq b+2 \alpha \pi .
$$

Using the fact that the dihedral angles sum to $2 \pi$, we find

$$
\sigma(H)+m \operatorname{sol}(H) \leq b,
$$

so octahedra $H$ with diagonals in the range $[2.716,2.81]$ satisfy the requisite bound. 
Summing inequality (16.6) over a consistently scored octahedron (either all compression or all vor) with diagonal in the range $[2.81,2 \sqrt{2}]$, we again arrive at the desired bound.

The remaining cases involve octahedra which contain both compression and vorscored quarters, and whose diagonals lie in the range $[2.81,2 \sqrt{2}]$. For this case we use inequality (16.7).

The summation involving inequality (16.7) is identical to inequality (16.6) save for the presence of the $\beta$ terms. If there are two vor-scored quarters and two compression-scored quarters, the beta terms cancel, giving the relation as before.

If there are three vor-scored quarters and one compression-scored quarter, we note that the same relation for vor-scored quarters holds if we replace $\beta$ by $\beta / 3$ (since we have now relaxed the bound). Summing the inequalities, the term involving $\beta$ vanishes again, leaving the desired inequality.

Lemmas 16.5 and 16.6 prove Proposition 15.5 for octahedra.

\subsection{Proof of Proposition 15.5: Pure Voronoi Quad Clusters}

The next class of quad clusters which we treat are the pure Voronoi quad clusters. We will define a truncation operation on these quad clusters. Truncation will simplify the geometry of the quad clusters, and will provide a convenient scoring bound. We divide our treatment of pure Voronoi quad clusters into two cases in order to simplify the analysis and numerical verifications as much as possible.

Recall from the classification of quad clusters that a pure Voronoi quad cluster consists of the intersection of a $V$-cell at the origin with the cone at the origin over a quadrilateral standard region. We refer to the restriction of the $V$-cell to the cone over the quadrilateral as either the $V$-cell or the Voronoi cell of the quad cluster. Figure 16.2 describes the geometry of a simple $V$-cell.

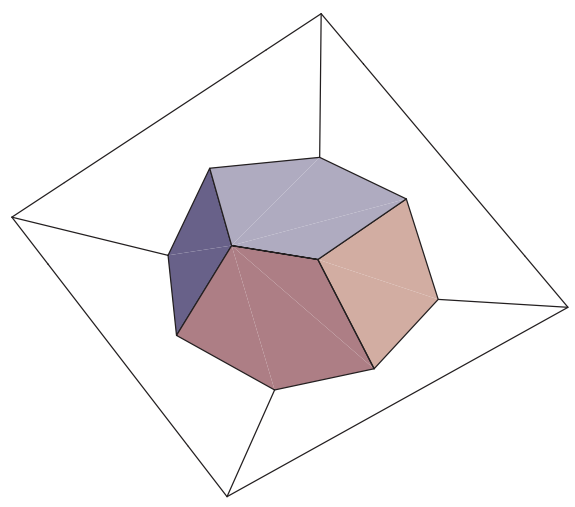

Fig. 16.2. A pure Voronoi quad cluster. 
In addition, recall that a vertex lying in the cone over a pure Voronoi quad cluster must have height greater than $2 \sqrt{2}$. Such vertices can significantly complicate the geometry of the $V$-cell, affecting its shape and volume.

We remove the effect of vertices lying above a pure Voronoi quad cluster by removing all points from the $V$-cell which have height greater than $\sqrt{2}$. We call this operation truncation at $\sqrt{2}$. Truncation decreases the volume of the quad cluster. This decrease in volume increases the score of the quad cluster, bringing it closer to the proposed bound.

We refer to truncated pure Voronoi quad clusters as truncated quad clusters.

We define a scoring operation on pure Voronoi quad clusters which we call truncated Voronoi scoring. This operation consists of truncation at $\sqrt{2}$, followed by the usual Voronoi scoring.

Each diagonal across the face of a cluster must have length greater than $2 \sqrt{2}$, otherwise we could form two flat quarters, contradicting the decomposition. We choose the shorter of the two possible diagonals, and consider that diagonal in the analysis which follows.

We decompose the cluster into two tetrahedrons along the chosen diagonal. The face dividing the tetrahedrons is either acute or it is obtuse. We treat each case separately.

We must prove

$$
\sigma(Q)+m \operatorname{sol}(Q)-b \leq 0,
$$

where $\sigma(Q)$ denotes the score of a pure Voronoi quad cluster $Q, \operatorname{sol}(Q)$ denotes the solid angle associated with the distinguished vertex, and $m$ and $b$ are positive constants. We call this relation a bound on the solid angle and score of a quad cluster. Invoking Proposition 15.2, we restrict our attention to quad clusters whose score exceeds $-1.04 \mathrm{pt}$.

\subsection{Pure Voronoi Quad Clusters: Acute Case}

Lemma 16.7. If an acute quad cluster is divided along an acute separating face, then the score of each half is nonpositive.

Proof. This is a consequence of the arguments of Theorem 8.4.

We therefore restrict our attention to halves whose score exceeds $-1.04 p t$, by Proposition 15.2.

If the separating face is acute, we prove

$$
\sigma\left(S_{i}\right)+m \operatorname{sol}\left(S_{i}\right)-b / 2 \leq 0
$$

for each half $S_{i}$ independently, and deduce the desired bound by adding the bounds for each half.

Lemma 16.8. Let $T_{0}$ denote the tetrahedron with edge lengths $(2,2,2,2,2,2 \sqrt{2})$. Let $\operatorname{sol}\left(T_{0}\right)$ denote the solid angle of the tetrahedron $T_{0}$. Given a tetrahedron $T$, if $\operatorname{sol}(T)<\operatorname{sol}\left(T_{0}\right)$, then relation (16.8) holds.

Proof. If $\operatorname{sol}(T)<\operatorname{sol}\left(T_{0}\right)$, then $m \operatorname{sol}(T)<m \operatorname{sol}\left(T_{0}\right)$, hence

$$
m \operatorname{sol}(T)-b / 2<m \operatorname{sol}\left(T_{0}\right)-b / 2 \leq 0
$$


and

$$
\sigma(T)+m \operatorname{sol}(T)-b / 2<\sigma(T) \leq 0,
$$

by Lemma 16.7 .

We therefore may restrict our attention to halves whose solid angle is at least $\operatorname{sol}\left(T_{0}\right)$. In addition, we restrict our attention to halves for which the dividing face is acute.

Lemma 16.9. The relation

$$
\sigma(T)+m \operatorname{sol}(T)-b / 2 \leq 0
$$

holds for a tetrahedron $T$ forming half of an acute quad cluster with score exceeding $-1.04 p t$.

Proof. The required verifications for each half of an acute quad cluster are somewhat difficult to achieve directly, so we subdivide into a number of different cases in an attempt to reduce the complexity of the calculations. First, we show that the bound holds for all halves whose diagonal is at least 2.84 (Calculation 17.4.4.1). Using this information, we then prove the bound everywhere but in a small corner cell (Calculation 17.4.4.2). We then restrict our attention to the small corner cell (Calculation 17.4.4.3). These computations involve the use of partial derivative information, and include the required boundary computations.

Invoking Lemma 16.9 for each half and adding them proves Proposition 15.5 for the acute case.

\subsection{Pure Voronoi Quad Clusters: Obtuse Case}

If the separating face is obtuse, the analysis becomes significantly harder. It is no longer possible to prove the desired bound on each half independently. The dimension of the full bound, even using the usual dimension-reduction techniques, is too high to make the verification tractable numerically. Therefore we adopt a different approach.

Using the dimension-reduction technique, we push each vertex along its edge until the distance from each vertex to the origin is 2 . We call the resulting quad cluster a squashed cluster. Observe that the solid angle of the cluster is unchanged, while the volume of the Voronoi cell has decreased, thereby increasing the score of the cluster.

Since the central face is still obtuse, the length of the diagonal after this perturbation must still exceed $2 \sqrt{2}$. Note, however, that the other edge lengths in the quad cluster can be as small as $4 / 2.51$.

The geometry of the $V$-cell of a squashed cluster, assuming that there is no truncation from vertices of the packing lying above the quad cluster, is that of Fig. 16.2. When the $V$-cell is truncated at $\sqrt{2}$ from the origin, two potential arrangements arise. In the first arrangement, the truncated region is connected, as in Fig. 16.3. In second potential arrangement, the truncated region is formed of two disjoint pieces, as in Fig. 16.4. 


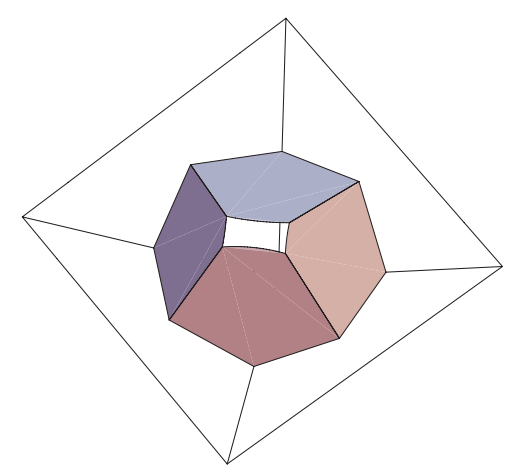

Fig. 16.3. A typical truncated quad cluster.

Lemma 16.10. The disjoint case cannot arise for squashed quad clusters.

Proof. Suppose that it could. Pick an untruncated point along the central ridge of the $V$-cell (see Fig. 16.4). The distance of this point from the origin is then less than $\sqrt{2}$, but due to its location on the central ridge, it is equidistant from the two nearest vertices and the origin. This implies that the circumradius of the resulting triangle must be less than $\sqrt{2}$, which contradicts the fact that the diagonals have length at least $2 \sqrt{2}$.

16.9.1. A Geometric Argument. We introduce a simplification which reduces the complexity of the obtuse case. This simplification consists of a perturbation of the upper edge lengths of a squashed quad cluster. This perturbation increases the score while holding the solid angle of the quad cluster fixed.

This simplification is based on a geometric decomposition of the truncated Voronoi cell. We describe the decomposition, and then describe a construction which will ultimately simplify the analysis.

While our arguments will extend to treat a general squashed and truncated Voronoi cell associated with a general standard cluster, we restrict our attention to truncated Voronoi cells associated with quad clusters.

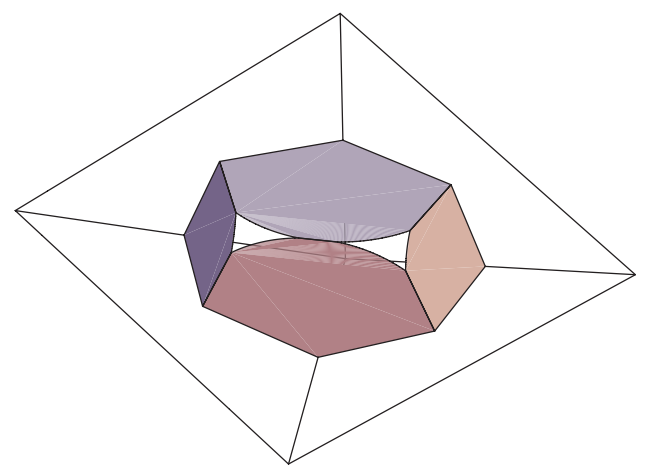

Fig. 16.4. An impossible arrangement. 


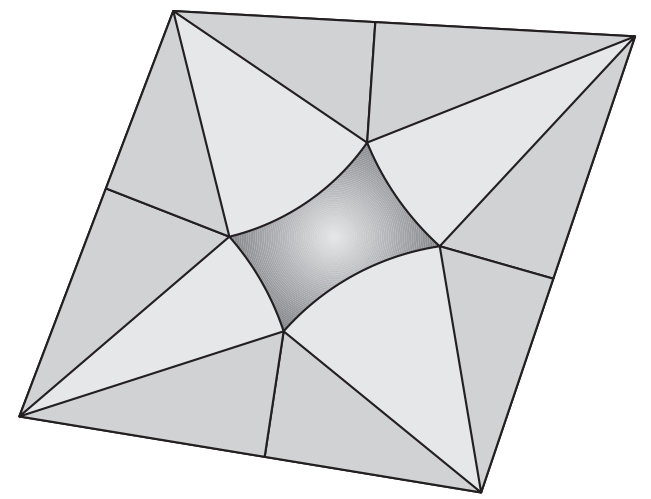

Fig. 16.5. A representation of a truncated quad cluster.

To begin, we consider the decomposition of a truncated Voronoi cell into its fundamental components. A truncated Voronoi cell is formed of three elements: a central spherical section (formed by the truncation), wedges of a right circular cone, and tetrahedrons called Rogers simplices.

We choose a representation of a truncated quad cluster composed of the radial projection of each element to a plane passing close to the four corners of the quad cluster. This decomposition is represented in Fig. 16.5.

16.9.2. Rogers Simplices. We now consider the geometry of the Rogers simplices.

Consider a face with edge lengths $(2,2, t)$ associated with a side of a truncated quad cluster. Let $b$ represent the circumradius of the face, and let $r$ represent the orthogonal extension of a Rogers simplex from the face, as in Fig. 16.6.

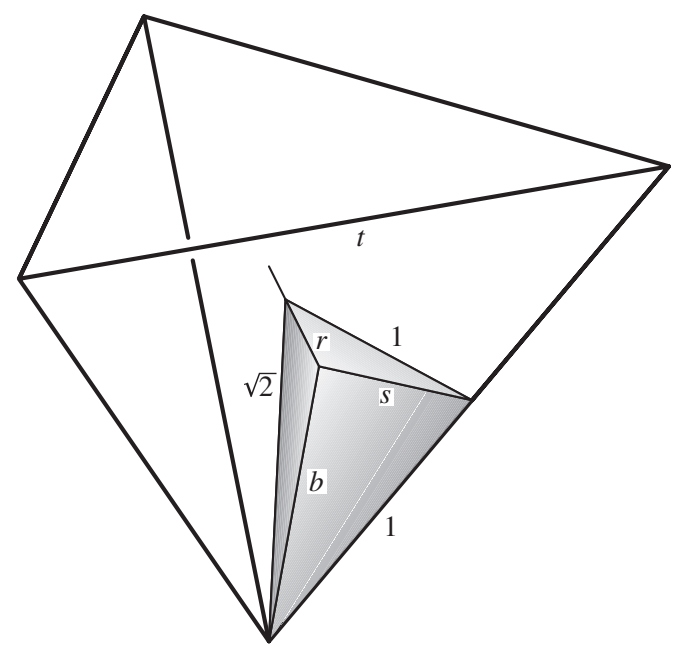

Fig. 16.6. Detail of truncated Voronoi decomposition. 


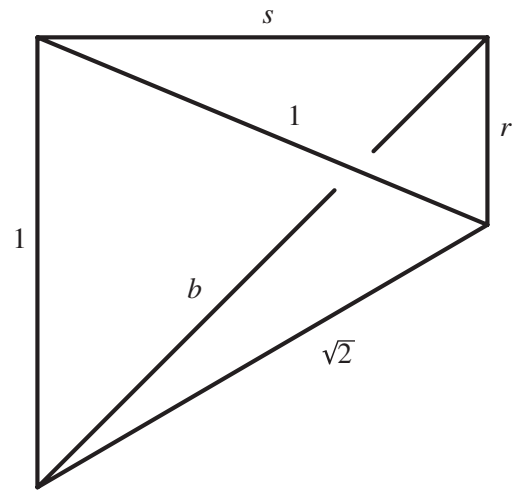

Fig. 16.7. Detail of Rogers simplex.

Then

$$
\begin{gathered}
b=\frac{4}{\sqrt{16-t^{2}}}, \\
r=\sqrt{2-b^{2}}=\sqrt{\frac{16-2 t^{2}}{16-t^{2}}},
\end{gathered}
$$

and

$$
s=\sqrt{b^{2}-1}=\frac{t}{\sqrt{16-t^{2}}} .
$$

See Fig. 16.7.

16.9.3. The Geometric Construction. We now present the geometric construction which implies the simplification.

We represent the geometry of the truncated Voronoi cell associated with one-half of a quad cluster in Fig. 16.8.

We can simplify the representation by extending the wedges to enclose the Rogers simplices. See Fig. 16.9. This process adds an extra volume term.

The overlap between the wedges is slightly complicated. We simplify the overlap as follows. Take the cone over the overlap. Intersect it with a ball of radius $\sqrt{2}$ at the origin. We call the spherical sections produced by this construction flutes. This construction is represented in Fig. 16.10. Figure 16.11 is a planar representation of this construction.

To form each flute, we have added two extra pieces of volume (per flute) to our construction. We call these pieces quoins. We attach each quoin to a Rogers simplex. See Fig. 16.12.

16.9.4. A Solid Angle Invariant. We now require some notation for the volumes which enter into this construction. Let $c$ denote the volume of the central spherical angle. Let $r$ denote the volume of the Rogers simplices. Let $w$ denote the volume of the wedges. Let $w^{\prime}$ denote the volume of the extended wedges. Let $q$ denote the volume of the quoins. 


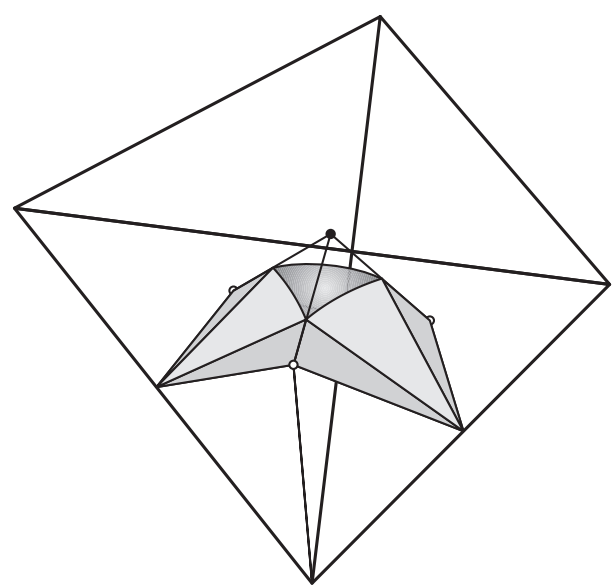

Fig. 16.8. Decomposition of a truncated Voronoi cell.

Let $f$ denote the volume of the flutes. Finally, let $v$ denote the volume of the truncated Voronoi cell.

Lemma 16.11. If we hold the solid angle fixed, the volume of a truncated squashed Voronoi cell depends only on $q$, the volume of the quoins.

Proof. By the original decomposition,

$$
v=c+r+w .
$$

By our construction,

$$
v=c+w^{\prime}+q-f .
$$

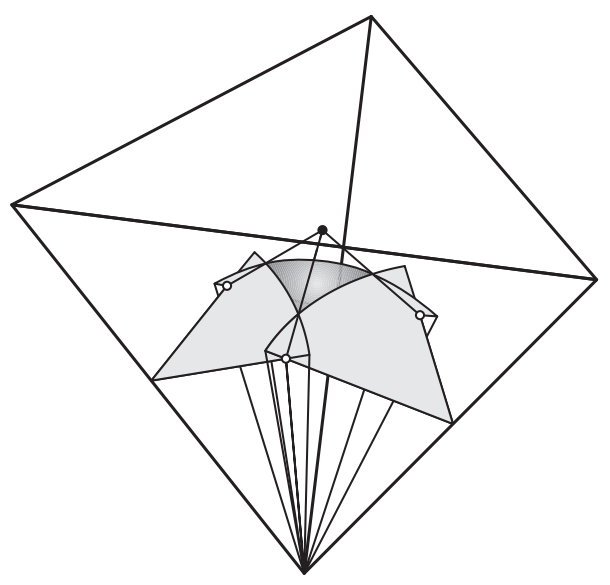

Fig. 16.9. Wedges extended to include the Rogers simplices. 


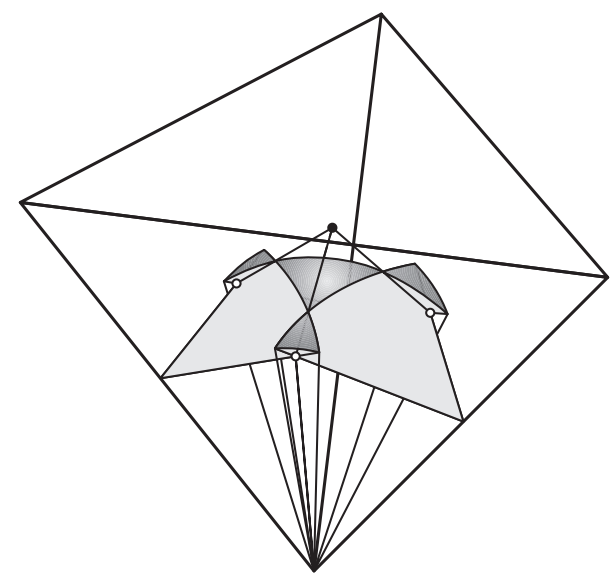

Fig. 16.10. Decomposition with flutes.

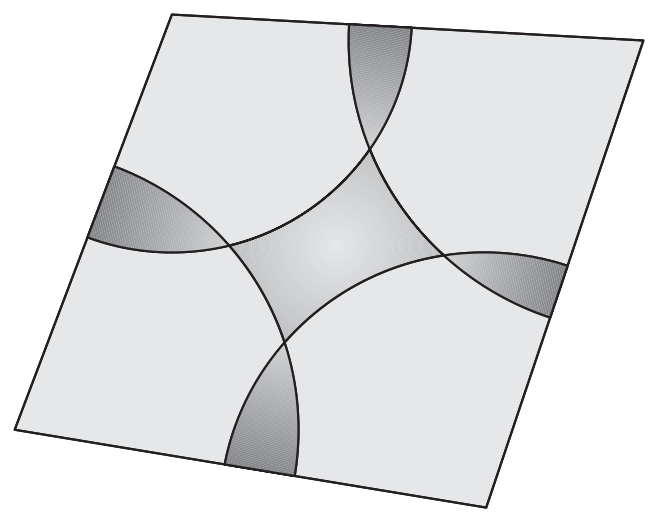

Fig. 16.11. Planar representation with flutes.

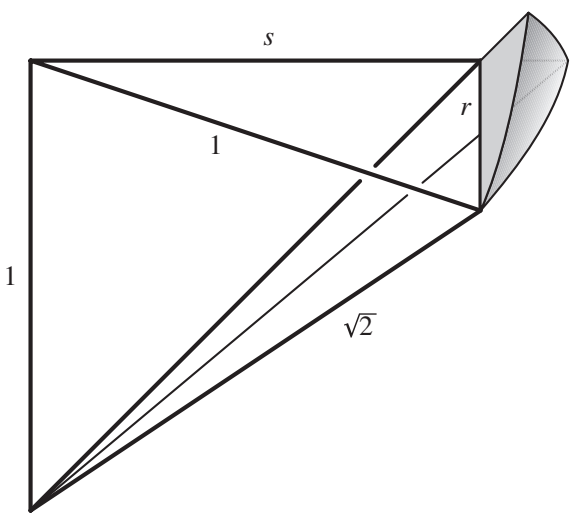

Fig. 16.12. Detail of Rogers simplex with quoin. 
Recall that the solid angle $s$ of the quad cluster is the sum of the dihedral angles minus $2 \pi$. The dihedral angles to which we refer are those associated with the edges between each corner of the quad cluster and the origin.

Our perturbation will hold the solid angle $s$ of the quad cluster fixed. Therefore, the sum of the dihedral angles must also be fixed. This fixes $w^{\prime}$.

Take the cone over each extended wedge and intersect it with a ball of radius $\sqrt{2}$ centered at the origin. Let $t$ denote the sum of these volumes. Since the sum of the dihedral angles is fixed, $t$ is also fixed.

Further, note that

$$
\frac{2 \sqrt{2}}{3} s=c+t-f \text {. }
$$

This relation implies that $c-f$ is fixed. Combining this with the previous relations, we find that if we hold the solid angle fixed, the volume of the truncated Voronoi cell depends only on $q$, the volume of the quoins.

16.9.5. Variation of the Volume of a Quoin. Consider a face $(2,2, t)$ of a truncated quad cluster. Two Rogers simplices are associated with this face, as suggested in Fig. 16.6. Observe that the volume of the quoin associated with one of these Rogers simplices is increasing in $r=\sqrt{\left(16-2 t^{2}\right) /\left(16-t^{2}\right)}$. Next, observe that $r$ is in turn decreasing in $t$. Therefore increasing $t$ decreases the volume of the squashed quad cluster, if we hold the solid angle fixed (by varying the length of another edge of the squashed quad cluster).

Each half of a squashed quad cluster has two variable edge lengths (not counting the shared diagonal). We label the variable edge lengths of one-half of the squashed quad cluster $y_{1}$ and $y_{2}$. We label the length of the diagonal $d$. Holding the solid angle fixed, we may perturb one half by shrinking the larger and increasing the shorter length. We wish to establish the following lemma, that increasing the short length reduces the volume of the truncated Voronoi cell more than decreasing the longer length increases the volume.

Lemma 16.12. Holding the solid angle fixed for one-half of a squashed quad cluster, shrinking the longer upper edge (while increasing the shorter edge appropriately) reduces the volume of the squashed quad cluster (increasing the score).

To prove Lemma 16.12, we establish a variational formula for the volume of a quoin.

We then verify that the volume of the quoin associated with the shorter edge is decreasing faster under this perturbation than the volume of the quoin associated with the longer edge is increasing.

In other words, we wish to show that $y_{1}<y_{2}$ implies that $V\left(y_{1}\right)+V\left(y_{2}\left(y_{1}\right)\right)$ is decreasing in $y_{1}$, or equivalently,

$$
V_{t}\left(y_{1}\right)+V_{t}\left(y_{2}\left(y_{1}\right)\right) \frac{d y_{2}}{d y_{1}}<0,
$$

where $V(t)$ is the volume of the quoin, $V_{t}(t)$ is the derivative of the volume, and $y_{2}$ is an implicit function of $y_{1}$.

We construct the volume of a quoin by integrating the area of a slice. We place the quoin in a convenient coordinate system. See Figs. 16.13 and 16.14. The truncating 


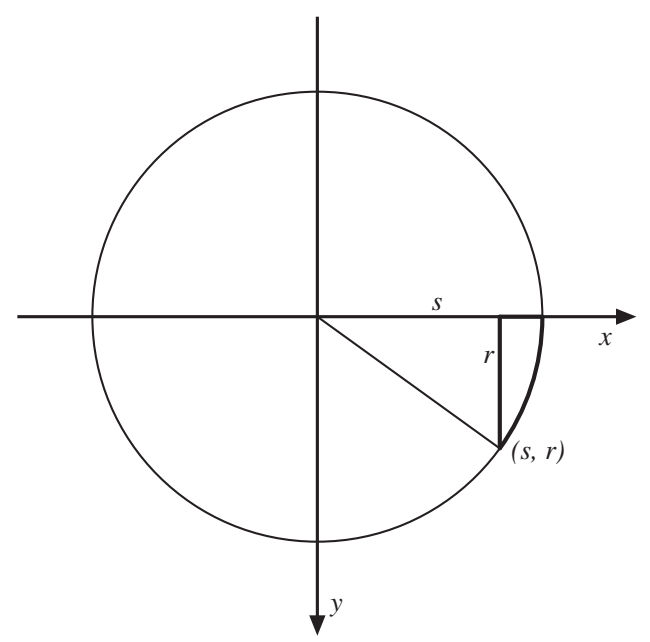

Fig. 16.13. Top view of quoin

sphere has equation $x^{2}+y^{2}+z^{2}=2$. At the base of the quoin, $z=1$, so $x=\sqrt{1-y^{2}}$ gives the location of the right-boundary of the quoin. The plane forming the left face of the quoin is given by the equation $x=s z$, so the ridge of the quoin is given by the curve $(s u, y, u)$, where $u=\sqrt{\left(2-y^{2}\right) /\left(1+s^{2}\right)}$.

Hence the area of a slice parallel to the $x-z$ plane is given by the formula

$$
A(t, y)=\frac{1}{2}(s u-s)(u-1)+\int_{s u}^{\sqrt{1-y^{2}}}\left(\sqrt{2-x^{2}-y^{2}}-1\right) d x .
$$

The volume of a quoin is therefore given by the formula

$$
V(t)=\int_{0}^{r} A(t, y) d y
$$

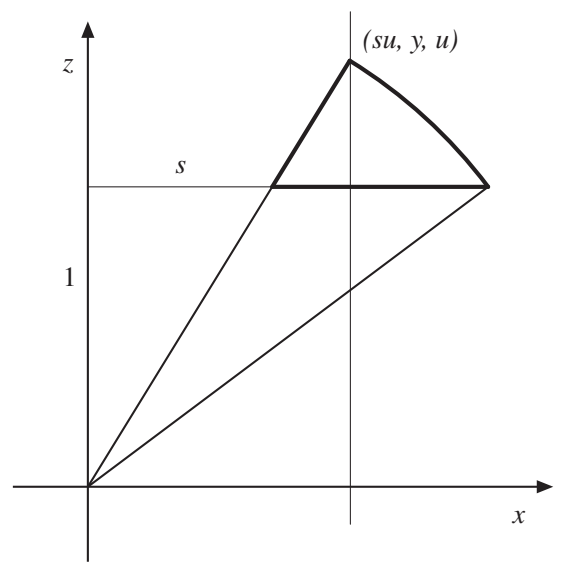

Fig. 16.14. Side view of quoin. 
We actually only need to compute $V_{t}(t)$, which is fortunate, since the explicit formula for $V(t)$ is somewhat complicated. We have

$$
V_{t}(t)=\int_{0}^{r} A_{t}(t, y) d y+A(t, r) r_{t},
$$

but $A(t, r)=0$, so

$$
V_{t}(t)=\int_{0}^{r} A_{t}(t, y) d y .
$$

So in addition, we only need $A_{t}(t, y)$,

$$
A_{t}(t, y)=\left(\frac{s}{2}\left(u^{2}+1\right)-\sqrt{1-y^{2}}+\int_{t / 4 \sqrt{2-y^{2}}}^{\sqrt{1-y^{2}}} \sqrt{2-x^{2}-y^{2}} d x\right)_{t},
$$

so

$$
A_{t}(t, y)=\left(\frac{s}{2}\left(u^{2}+1\right)\right)_{t}-\sqrt{2-\frac{t^{2}}{16}\left(2-y^{2}\right)-y^{2}} \frac{1}{4} \sqrt{2-y^{2}},
$$

which simplifies to

$$
A_{t}(t, y)=\frac{8}{\left(16-t^{2}\right)^{3 / 2}}-\frac{2-y^{2}}{2 \sqrt{16-t^{2}}}
$$

Hence

$$
V_{t}(t)=\frac{8 r}{\left(16-t^{2}\right)^{3 / 2}}-\frac{r}{\sqrt{16-t^{2}}}+\frac{r^{3}}{6 \sqrt{16-t^{2}}},
$$

which simplifies to

$$
V_{t}(t)=\frac{-2 \sqrt{2}\left(8-t^{2}\right)^{3 / 2}}{3\left(16-t^{2}\right)^{2}} .
$$

We are now prepared to prove Lemma 16.12 .

Proof. Holding the solid angle fixed, $y_{2}$ is an implicit function of $y_{1}$. We wish to prove that $y_{1}<y_{2}$ implies

$$
V_{t}\left(y_{1}\right)+V_{t}\left(y_{2}\right) \frac{d y_{2}}{d y_{1}}<0 .
$$

We derive a formula for $d y_{2} / d y_{1}$, using the solid angle constraint

$$
\operatorname{sol}\left(2,2,2, y_{1}, y_{2}, d\right)=c,
$$

where $c$ is a constant. Using formulas from [Ha6], (16.10) becomes

$$
2 \arctan \left(\frac{\sqrt{\Delta}}{2 a}\right)=c .
$$


Let $x_{1}=y_{1}^{2}, x_{2}=y_{2}^{2}$, and $b=d^{2}$. Then

$$
\Delta=-4 b^{2}-4\left(x_{1}-x_{2}\right)^{2}+b\left(x_{1}\left(8-x_{2}\right)+8 x_{2}\right)
$$

and

$$
a=32-d-x_{1}-x_{2} .
$$

So

$$
\frac{-4 b^{2}-4\left(x_{1}-x_{2}\right)^{2}+b\left(x_{1}\left(8-x_{2}\right)+8 x_{2}\right)}{\left(32-d-x_{1}-x_{2}\right)^{2}}=c_{1} .
$$

Therefore

$$
\frac{d x_{2}}{d x_{1}}=-\frac{\left(16-x_{2}\right)\left(x_{2}+b-x_{1}\right)}{\left(16-x_{1}\right)\left(x_{1}+b-x_{2}\right)}
$$

and

$$
\frac{d y_{2}}{d y_{1}}=\frac{y_{1}}{y_{2}} \frac{d x_{2}}{d x_{1}}
$$

hence

$$
\frac{d y_{2}}{d y_{1}}=-\frac{y_{1}\left(16-x_{2}\right)\left(x_{2}+b-x_{1}\right)}{y_{2}\left(16-x_{1}\right)\left(x_{1}+b-x_{2}\right)} .
$$

We substitute the formula for $d y_{2} / d y_{1}$ into (16.9). Letting $x_{i}=y_{i}^{2}$, and noting that all the denominators are positive, we obtain on clearing denominators that the desired relation (16.9) is equivalent to

$-\left(8-x_{1}\right)^{3 / 2}\left(16-x_{2}\right) y_{2}\left(x_{1}+b-x_{2}\right)+\left(8-x_{2}\right)^{3 / 2}\left(16-x_{1}\right) y_{1}\left(x_{2}+b-x_{1}\right)<0$,

or

$$
\left(16-x_{1}\right)^{2} x_{1}\left(8-x_{2}\right)^{3}\left(b-x_{1}+x_{2}\right)^{2}<\left(16-x_{2}\right)^{2} x_{2}\left(8-x_{1}\right)^{3}\left(b+x_{1}-x_{2}\right)^{2} .
$$

If we define

$$
g\left(x_{1}, x_{2}\right)=\left(16-x_{1}\right)^{2} x_{1}\left(8-x_{2}\right)^{3}\left(b-x_{1}+x_{2}\right)^{2},
$$

then the desired inequality is equivalent to $g\left(x_{1}, x_{2}\right)<g\left(x_{2}, x_{1}\right)$ for $x_{1}<x_{2}$. There are several ways to prove this monotonicity relation. One is to prove that the polynomial

$$
\frac{g\left(x_{1}, x_{2}\right)-g\left(x_{2}, x_{1}\right)}{8\left(x_{1}-x_{2}\right)}
$$

is positive for all allowable values for $x_{1}, x_{2}$, and $b$. Unfortunately, the resulting polynomial has degree 6 , so the verification is somewhat unwieldy, although easy enough using interval methods.

A simpler method involves a factorization of $g$ into $g_{1}$ and $g_{2}$. We show that $g_{1}$ and $g_{2}$ each satisfy the monotonicity relation, and the relation then follows for $g$.

Define

$$
g_{1}\left(x_{1}, x_{2}\right)=\left(16-x_{1}\right) x_{1}\left(8-x_{2}\right)\left(b-x_{1}+x_{2}\right)
$$


and

$$
g_{2}\left(x_{1}, x_{2}\right)=\left(16-x_{1}\right)\left(8-x_{2}\right)^{2}\left(b-x_{1}+x_{2}\right) .
$$

Clearly $g=g_{1} g_{2}$. We then construct the polynomials

$$
p_{1}=\frac{g_{1}\left(x_{1}, x_{2}\right)-g_{1}\left(x_{2}, x_{1}\right)}{x_{1}-x_{2}}
$$

and

$$
p_{2}=\frac{g_{2}\left(x_{1}, x_{2}\right)-g_{2}\left(x_{2}, x_{1}\right)}{x_{1}-x_{2}} .
$$

Simplifying $p_{1}$ and $p_{2}$, we find that

$$
\begin{aligned}
p_{1}= & 128 b-128 x_{1}-8 b x_{1}+8 x_{1}^{2}-128 x_{2} \\
& +32 x_{1} x_{2}+b x_{1} x_{2}-x_{1}^{2} x_{2}+8 x_{2}^{2}-x_{1} x_{2}^{2}
\end{aligned}
$$

and

$$
\begin{aligned}
p_{2}= & -2048+192 b+320 x_{1}-16 b x_{1}-16 x_{1}^{2}+320 x_{2} \\
& -16 b x_{2}-32 x_{1} x_{2}+b x_{1} x_{2}+x_{1}^{2} x_{2}-16 x_{2}^{2}+x_{1} x_{2}^{2} .
\end{aligned}
$$

These polynomials are quadratic in $x_{1}$ and $x_{2}$, and linear in $b$. The coefficient of $b$ in $p_{1}$ is

$$
128-8 x_{1}-8 x_{2}+x_{1} x_{2} .
$$

The coefficient of $b$ in $p_{2}$ is

$$
192-16 x_{1}-16 x_{2}+x_{1} x_{2} .
$$

Both coefficients are positive for $x_{1}$ and $x_{2}$ in $\left[16 / 2.51^{2}, 2.51^{2}\right]$. Therefore, the minimum values of $p_{1}$ and $p_{2}$ occur when $b$ is at a minimum, $b=8$.

The minimum value of each polynomial for values of $x_{1}$ and $x_{2}$ in the range $\left[16 / 2.51^{2}, 2.51^{2}\right]$ is now easily computed. Making the appropriate computations, we find that each polynomial is indeed positive. Hence the desired relation follows.

\subsubsection{Final Simplification.}

Lemma 16.13. Obtuse quad clusters satisfy the bound of Proposition 15.5.

Proof. We begin with a squashed quad cluster with consecutive upper edge lengths $\left(y_{1}, y_{2}, y_{3}, y_{4}\right)$ and diagonal $d$ adjacent to the first two upper edges.

Recall that we chose the diagonal of the quad cluster to be the shorter of the two possible diagonals. We refer to the other possible diagonal as the cross-diagonal. Recall that the reduction fixes the length of the diagonal.

If the length of the cross-diagonal does not drop to $2 \sqrt{2}$ under the perturbation of Lemma 16.12, we arrive at the configuration with edge lengths $\left(y_{1}^{\prime}, y_{1}^{\prime}, y_{2}^{\prime}, y_{2}^{\prime}\right)$ with diagonal $d$. 
If the length of the cross-diagonal does drop to $2 \sqrt{2}$, then stop the perturbation. This gives a quadrilateral $\left(y_{1}^{\prime}, y_{2}^{\prime}, y_{3}^{\prime}, y_{4}^{\prime}\right)$ with diagonal $2 \sqrt{2}$. Applying the perturbation to each half independently, we find that the score of each half is maximized by the configuration $\left(y_{1}^{\prime \prime}, y_{1}^{\prime \prime}, y_{2}^{\prime \prime}, y_{2}^{\prime \prime}\right)$ with diagonal $2 \sqrt{2}$. We verify the relation for this arrangement in Calculation 17.4.4.5.

If the length of the cross-diagonal did not drop to $2 \sqrt{2}$, switch to the cross-diagonal and repeat the process. If the (new) cross-diagonal does not drop to $2 \sqrt{2}$, we have arrived at the configuration $(y, y, y, y)$ with diagonal $d^{\prime}$. Choose a new diagonal $d^{\prime \prime}$ to be the shorter of the two possible diagonals. We verify the desired relation for this arrangement in Calculation 17.4.4.4.

Finally, we make a few comments about extra constraints in the verifications.

Since the score of a quad cluster is nonpositive, and $m\left(2 \operatorname{sol}\left(T_{0}\right)\right)-b \leq 0$ where $\operatorname{sol}\left(T_{0}\right)=\operatorname{sol}(2,2,2,2,2,2 \sqrt{2})$, we need only consider quad clusters for which the solid angle exceeds $2 \operatorname{sol}\left(T_{0}\right)$.

The maximum length of the diagonal is $2.51 \sqrt{2}$, since otherwise the triangles in the quadrilateral would be obtuse, forcing the cross-diagonal to be shorter than the diagonal. This would contradict our original choice of the shortest diagonal.

In Calculation 17.4.4.4 we assume that $d$ is the shortest diagonal. Adding this constraint directly is tedious, since the formula for the cross-diagonal of the quad cluster is somewhat complicated. We apply a simpler but weaker constraint, that the diagonal $d$ of a planar quadrilateral with edge lengths $(y, y, y, y)$ is shorter than $d^{\prime}$, the other planar diagonal. The constraint $d \leq d^{\prime}$ gives the constraint $d^{2} \leq 2 y^{2}$. Since the cross-diagonal of the quad cluster is shorter than the cross-diagonal of the planar quadrilateral, this constraint is weaker.

Lemmas 16.9 and 16.13 prove Proposition 15.5 for pure Voronoi quad clusters.

\section{Calculations}

The verifications of the relations required in this paper appear intractable using traditional methods. Therefore, we use a relatively new proof technique, interval arithmetic via floating-point computer calculations.

\subsection{Interval Arithmetic}

We review the basic notions of interval arithmetic.

Suppose that the value of a function $f(x)$ lies in the interval $[a, b]$. Further, suppose that $g(x)$ lies in the interval $[c, d]$. Then $f(x)+g(x)$ must lie in $[a+c, b+d]$. While it may be the case that we could produce better bounds than this for the function $f+g$, these interval bounds give crude control over the behavior of the function. Interval arithmetic provides a mechanism for formalizing arithmetic on these bounds.

We represent an interval $t$ as $[\underline{t}, \bar{t}]$. Then for intervals $a$ and $b$,

$$
a+b=[\underline{a}+\underline{b}, \bar{a}+\bar{b}] \text {. }
$$


Likewise,

$$
a-b=[\underline{a}-\bar{b}, \bar{a}-\underline{b}] .
$$

Multiplication is somewhat more complicated. Define

$$
C=\{\underline{a b}, \underline{a} \bar{b}, \bar{a} \underline{b}, \bar{a} \bar{b}\} .
$$

Then

$$
a * b=[\min (C), \max (C)] .
$$

Division is similar, as long as the dividing interval does not contain zero.

Similarly, we can define the operation of a monotonic function on an interval. For example,

$$
\arctan (a)=[\arctan (\underline{a}), \arctan (\bar{a})] .
$$

Using interval arithmetic, we can produce rigorous bounds for polynomials evaluated on intervals. Likewise, we can produce rigorous bounds for rational functions evaluated on intervals. Finally, we add the composition of monotonic functions. This allows us to produce interval bounds for functions such as $\operatorname{sol}(\cdot)$ and vor(.) over quasi-regular tetrahedrons, quarters, or quad clusters.

\subsection{The Method of Subdivision}

The relations on tetrahedra and quad clusters required for our approach typically have the form $g(y) \leq 0$ for $y \in I$, where $I$ is a product of closed intervals. As $g$ is usually continuous, the existence of a maximum is trivial. However, bounds on the behavior of $g$ over all of $I$ computed directly via interval arithmetic are generally poor.

We define a cell to be a product of closed intervals. By subdividing $I$ into sufficiently small cells, the quality of the computed bounds on each cell usually improves enough to prove the relation for each cell, and hence for the original domain $I$.

If in fact $g(y) \leq c<0$, this approach works very well. However, if the bound is tight at a point $y_{0}$, i.e., $g\left(y_{0}\right)=0$, then pure subdivision will usually fail, since the computed upper bound on $g$ over any cell containing $y_{0}$ will typically be positive.

If $y_{0}$ is not an interior maximum, we turn to the partial derivatives of $g$. If we can show that the partials of $g$ on a small cell containing $y_{0}$ have fixed sign (bounded away from zero), then the maximum value of $g$ on that cell is easily computed. It is typically the case that a cell must be very small before we can determine the sign of the partials via interval-arithmetic bounds.

\subsection{Numerical Considerations}

Most real numbers are not representable in computer floating-point format. However, floating-point intervals may be found which contain any real number. Although the magnitude of real numbers representable in fixed-length floating-point format is finite, the format also provides for $\pm \infty$, which allows for interval containment of all reals. 
These intervals may be added, multiplied, etc., and the resulting intervals will contain the result of the operation applied to the real numbers which they represent.

Since floating-point arithmetic is not exact, interval arithmetic conducted using floating-point arithmetic is not optimal, in the sense that the interval resulting from an operation will usually be larger than the true resultant interval, due to roundoff. However, barring hardware or software errors (implementation errors, not roundoff errors), floating-point interval arithmetic, unlike floating-point arithmetic, is correct, in the sense that it provides correct interval bounds on the value of a computation, while floating-point arithmetic alone only provides an approximation to the correct value of a computation. We may therefore use interval arithmetic to prove mathematical results. Floating-point arithmetic alone, in the absence of rigorous error analysis, cannot constitute a proof.

We implement floating-point interval arithmetic routines via the IEEE 754 Standard for floating-point arithmetic [IEEE].

Implementation of interval arithmetic is straightforward using directed rounding. In addition to arithmetic functions, we require interval implementations of the square root and arctangent functions. Fortunately, the IEEE standard provides the square root function. However, the arctangent function is somewhat problematic, since the standard mathematical libraries do not provide explicit error bounds for their implementations of the arctangent function. In theory, they should provide an accuracy for the arctangent routine of $0.7 \mathrm{ulps}$, meaning that the error is less than one unit in the last place. I add interval padding of the form $[v-\varepsilon, v+\varepsilon]$, where $v$ is the computed value, and $\varepsilon=2^{-49}$. This should be sufficient to guarantee proper interval containment, assuming that the library routines are correctly implemented.

Armed with standard interval arithmetic and interval arithmetic implementations of sqrt and arctan, we can implement interval arithmetic versions of all the special functions required for proving the sphere packing relations.

Evaluating these functions on cells, we get bounds. Unfortunately, these bounds are not very good. The bounds which we get from interval versions of the partial derivative functions are even worse. This means that cells have to be very small before we can draw conclusions about the signs of the partials. These bad bounds are due to the inherent nature of interval arithmetic - it produces worst-case results by design.

These bad bounds increase the complexity of the verifications tremendously. Some verifications, using these bounds, require the consideration of billions or trillions of cells, or worse. Therefore, we needed a method for producing better bounds than those which direct interval methods could provide.

The method which we eventually discovered is to use Taylor series. We compute explicit second (mixed) partial bounds for the major special functions, and use these bounds to produce very good interval bounds. These bounds are computed in Calculations 17.4.6.1-17.4.6.8. Essentially, the Taylor method postpones the error bound until the end of the computation, eliminating the error bound explosion which occurs with a straightforward interval method implementation.

\subsection{Calculations}

The following inequalities have been proved by computer using interval methods. Let $S=S(y)=S\left(y_{1}, \ldots, y_{6}\right)$ denote a tetrahedron parametrized by the edge lengths 
$\left(y_{1}, \ldots, y_{6}\right)$. In addition, we often parametrize by the squares of the edge lengths $\left(x_{1}, \ldots, x_{6}\right)$.

Recall from Section 15.1 that $m=0.3621, b=0.49246, a=0.0739626$, and $b_{c}=0.253095$.

Recall that for our purposes, the scoring function $\sigma(\cdot)$ is given by one of four functions: $g m a(\cdot), \operatorname{vor}(\cdot)$, octavor $(\cdot)$, or truncated Voronoi. See Remark 7.23 for a simplified version of the scoring function.

The scoring rules depend on $\eta(\cdot)$, the circumradius of a face, introduced in Definition 4.20.

17.4.1. Quasi-Regular Tetrahedra. Define $C=[2,2.51]^{6}$, and recall

$$
a_{1}=0.3860658808124052, \quad a_{2}=0.4198577862, \quad d_{0}=1.4674 .
$$

Calculation 17.4.1.1. Either

$$
g m a(S) \leq a_{1} \operatorname{dih}(S)-a_{2}
$$

or

$$
\operatorname{gma}(S) \leq-0.52 p t
$$

for $y \in C$, using dimension-reduction.

Calculation 17.4.1.2. Either

$$
g m a(S)-a_{1} \operatorname{dih}(S) \leq 3.48 p t-2 \pi a_{1}+4 a_{2}
$$

or

$$
\operatorname{dih}(S)<d_{0}
$$

or

$$
\operatorname{gma}(S) \leq-0.52 p t
$$

for $y \in C$, using dimension-reduction.

Calculation 17.4.1.3. Either

$$
g m a(S)+m \operatorname{sol}(S)+a(\operatorname{dih}(S)-2 \pi / 5)-b_{c} \leq 0
$$

or

$$
\operatorname{dih}(S)>d_{0}
$$

or

$$
\operatorname{gma}(S) \leq-0.52 p t
$$

for $y \in C$, using dimension-reduction. 
17.4.2. Flat Quad Clusters. Define $I=[2,2.51]^{5}[2.51,2 \sqrt{2}]$, and define the corner cell

$$
C=[2,2+0.51 / 16]^{5}[2 \sqrt{2}-(2 \sqrt{2}-2.51) / 16,2 \sqrt{2}] .
$$

Calculation 17.4.2.1. Either

$$
g m a(S)+m \operatorname{sol}(S) \leq b / 2
$$

or

$$
\eta\left(y_{1}, y_{2}, y_{6}\right)^{2}>2
$$

or

$$
\eta\left(y_{4}, y_{5}, y_{6}\right)^{2}>2
$$

or

$$
\operatorname{gma}(S) \leq-1.04 p t
$$

for $y \in I$, using dimension reduction.

Calculation 17.4.2.2. Either

$$
g m a(S)+m \operatorname{sol}(S) \leq b / 2
$$

or

$$
\eta\left(y_{1}, y_{2}, y_{6}\right)^{2}=2 \quad \text { with } \quad \eta\left(y_{4}, y_{5}, y_{6}\right)^{2} \leq 2
$$

or

$$
\eta\left(y_{4}, y_{5}, y_{6}\right)^{2}=2 \quad \text { with } \quad \eta\left(y_{1}, y_{2}, y_{6}\right)^{2} \leq 2
$$

or

$$
\operatorname{gma}(S) \leq-1.04 p t
$$

for $y \in I$, not using dimension-reduction.

Calculation 17.4.2.3. $d \operatorname{vor}(S) / d y_{i}<0$ for $i=1,2,3$ and $y \in C$.

Calculation 17.4.2.4. This computation is somewhat tricky, since the scoring constraint depends on both faces. The partial derivative information gives $y_{3}=2$. The rest of the analysis depends on which face is assumed to be large.

If the $\left(y_{1}, y_{2}, y_{6}\right)$ face is large, the partial derivative information implies that the face constraint is tight, so $\eta\left(y_{1}, y_{2}, y_{6}\right)^{2}=2$. Therefore solve for $y_{1}$ in terms of $y_{2}$ and $y_{6}$. Apply partial derivative information for $y_{4}$ and $y_{5}$. In this case,

$$
\operatorname{vor}(S)+m \operatorname{sol}(S) \leq b / 2
$$

for $y_{3}=2, y \in C$. 
If the $\left(y_{4}, y_{5}, y_{6}\right)$ face is large, assume that $y_{1}=y_{2}=2$. Then either

$$
\operatorname{vor}(S)+m \operatorname{sol}(S) \leq b / 2
$$

or

$$
\eta\left(y_{4}, y_{5}, y_{6}\right)^{2}<2
$$

for $y_{1}=y_{2}=y_{3}=2, y \in C$.

Calculation 17.4.2.5. Either

$$
\operatorname{vor}(S)+m \operatorname{sol}(S) \leq b / 2
$$

or

$$
\eta\left(y_{1}, y_{2}, y_{6}\right)^{2}<2 \text { and } \eta\left(y_{4}, y_{5}, y_{6}\right)^{2}<2
$$

or

$$
\operatorname{vor}(S) \leq-1.04 p t
$$

for $y \in I, y \notin C$, using dimension-reduction and partial derivative information.

Calculation 17.4.2.6. Either

$$
\operatorname{vor}(S)+m \operatorname{sol}(S) \leq b / 2,
$$

with

$$
\eta\left(y_{1}, y_{2}, y_{6}\right)^{2}=2 \text { or } \eta\left(y_{4}, y_{5}, y_{6}\right)^{2}=2 \text {, }
$$

or

$$
\operatorname{vor}(S) \leq-1.04 p t
$$

for $y \in I, y \notin C$, not using dimension-reduction.

\subsubsection{Octahedra}

Calculation 17.4.3.1. $\sigma(S) \leq-0.52$ pt, for each (appropriately scored) upright quarter with edge lengths in the cell $[2.51,2 \sqrt{2}][2.2,2.51][2,2.51]^{4}$.

Calculation 17.4.3.2. Recall $c=0.1533667634670977$ and $d=0.2265$. Either

$$
\operatorname{gma}(S)+c \operatorname{dih}(S) \leq d
$$

or

$$
\operatorname{gma}(S) \leq-1.04 p t
$$

for $y \in[2.51,2.716][2,2.2]^{5}$, using dimension-reduction. Note that for both faces adjacent to the diagonal,

$$
\max \eta^{2}=\eta(2.2,2.2,2.716)^{2}<2,
$$

so all quarters in this cell are compression-scored. 
Calculation 17.4.3.3. Either

$$
g m a(S)+m \operatorname{sol}(S)+\alpha \operatorname{dih}(S) \leq \frac{b}{4}+\alpha \frac{\pi}{2}
$$

or

$$
\operatorname{gma}(S) \leq-1.04 p t
$$

for all compression-scored quarters $S(y)$, where $\alpha=0.14$,

$$
y \in[2.716,2 \sqrt{2}][2,2.2]^{2}[2,2.51][2,2.2]^{2},
$$

using dimension-reduction.

Calculation 17.4.3.4. Either

$$
\operatorname{octavor}(S)+m \operatorname{sol}(S)+\alpha \operatorname{dih}(S) \leq \frac{b}{4}+\alpha \frac{\pi}{2}
$$

or

$$
\operatorname{octavor}(S) \leq-1.04 p t
$$

for all vor analytic-scored quarters $S(y)$, where $\alpha=0.14$,

$$
y \in[2.716,2.81][2,2.2]^{2}[2,2.51][2,2.2]^{2} .
$$

Calculation 17.4.3.5. Either

$$
g m a(S)+m \operatorname{sol}(S)+\alpha \operatorname{dih}(S)+\beta x_{1} \leq \frac{b}{4}+\alpha \frac{\pi}{2}+8 \beta
$$

or

$$
\operatorname{gma}(S) \leq-1.04 p t
$$

for all compression-scored quarters $S(y)$, where $\alpha=0.054, \beta=0.00455, x_{1}=y_{1}^{2}$, and

$$
y \in[2.81,2 \sqrt{2}][2,2.2]^{2}[2,2.51][2,2.2]^{2},
$$

using some dimension-reduction.

Calculation 17.4.3.6. Either

$$
\operatorname{octavor}(S)+m \operatorname{sol}(S)+\alpha \operatorname{dih}(S)+\beta x_{1} \leq \frac{b}{4}+\alpha \frac{\pi}{2}+8 \beta
$$

or

$$
\operatorname{octavor}(S) \leq-1.04 p t
$$

for all vor analytic-scored quarters $S(y)$, where $\alpha=0.054, \beta=-0.00455, x_{1}=y_{1}^{2}$, and

$$
y \in[2.81,2 \sqrt{2}][2,2.2]^{2}[2,2.51][2,2.2]^{2} .
$$


17.4.4. Pure Voronoi Quad Clusters. Recall sol( $\left(T_{0}\right)$ denotes the solid angle of the tetrahedron $(2,2,2,2,2,2 \sqrt{2})$.

Define the corner cell $C=[2,2+0.51 / 8]^{5}[2 \sqrt{2}, 2.84]$. We denote truncated Voronoi scoring by $\sigma$. The constraint that the dividing face be acute translates into $x_{1}+x_{2}-x_{6} \geq 0$. In each computation we apply dimension-reduction.

We begin with the acute case.

Calculation 17.4.4.1. Either

$$
\sigma(S)+m \operatorname{sol}(S)-b / 2 \leq 0
$$

or

$$
\operatorname{sol}(S)<\operatorname{sol}\left(T_{0}\right)
$$

or

$$
x_{1}+x_{2}-x_{6}<0
$$

or

$$
\sigma(S) \leq-1.04 p t
$$

for $y \in[2,2.51]^{5}[2.84,4]$.

Calculation 17.4.4.2. Either

$$
\sigma(S)+m \operatorname{sol}(S)-b / 2 \leq 0
$$

or

$$
\operatorname{sol}(S)<\operatorname{sol}\left(T_{0}\right)
$$

or

$$
x_{1}+x_{2}-x_{6}<0
$$

or

$$
\sigma(S) \leq-1.04 p t
$$

for $y \in[2,2.51]^{5}[2 \sqrt{2}, 2.84]$ with $y \notin C$.

Calculation 17.4.4.3. Either

$$
\sigma(S)+m \operatorname{sol}(S)-b / 2 \leq 0
$$

or

$$
\operatorname{sol}(S)<\operatorname{sol}\left(T_{0}\right)
$$

or

$$
x_{1}+x_{2}-x_{6}<0,
$$

$y \in C$. 
Finally, we consider the obtuse case.

Calculation 17.4.4.4. Either

$$
\sigma(S)+m \operatorname{sol}(S)-b / 2 \leq 0
$$

or

$$
\operatorname{sol}(S)<\operatorname{sol}\left(T_{0}\right)
$$

or

$$
\sigma(S) \leq-0.52 p t
$$

or

$$
2 y^{2}<d^{2}
$$

for a symmetric pure Voronoi quad cluster composed of two copies of $S$, where

$$
S=(2,2,2, y, y, d)
$$

$y \in[4 / 2.51,2.51]$ and $d \in[2 \sqrt{2}, 2.51 \sqrt{2}]$.

Calculation 17.4.4.5. Either

$$
\sigma\left(S_{1}\right)+\sigma\left(S_{2}\right)+m\left(\operatorname{sol}\left(S_{1}\right)+\operatorname{sol}\left(S_{2}\right)\right)-b \leq 0
$$

or

$$
\sigma\left(S_{1}\right)+\sigma\left(S_{2}\right) \leq-1.04 p t
$$

or

$$
\operatorname{sol}\left(S_{1}\right)+\operatorname{sol}\left(S_{2}\right)<2 \operatorname{sol}\left(T_{0}\right)
$$

for a pure Voronoi quad cluster composed of two tetrahedrons $S_{1}$ and $S_{2}$, where

$$
S_{i}=\left(2,2,2, y_{i}, y_{i}, 2 \sqrt{2}\right)
$$

$y_{i} \in[4 / 2.51,2.51]$.

\subsubsection{Dimension Reduction}

Calculation 17.4.5.1. The polynomial derived for the dimension-reduction argument is positive for $x \in\left[4,2.51^{2}\right]^{6}$ and $x \in\left[4,2.51^{2}\right]^{5}[4,8]$. 
17.4.6. Second Partial Bounds. We compute all second partials $d^{2} / d x_{i} d x_{j}$ in terms of $x_{i}$, the squares of the edge lengths. We do each computation twice, once for quasiregular tetrahedrons and once for quarters. We compute the second partials of dih(.), sol(.), compression volume, and Voronoi volume (the vor analytic volume). Since the scoring functions are linear combinations of sol( $\cdot)$ and the volume terms, we may derive second partial bounds for $\operatorname{gma}(\cdot)$ and vor( $(\cdot)$ from these.

With the application of additional computer power, these bounds could be improved. These bounds were computed using sixteen subdivisions. While using thirty-two subdivisions would improve the bounds by a factor of 2, perhaps, the time required for the computations increases by a factor of 64 .

Calculation 17.4.6.1. For quasi-regular tetrahedrons $T$, the second partials of $\operatorname{dih}(T)$ lie in

$$
\text { [-0.0926959464, 0.0730008897]. }
$$

Calculation 17.4.6.2. For quarters $Q$, the second partials of $\operatorname{dih}(Q)$ lie in

$$
\text { [-0.2384125007, 0.169150875]. }
$$

Calculation 17.4.6.3. For quasi-regular tetrahedrons $T$, the second partials of $\operatorname{sol}(T)$ lie in

$$
[-0.0729140255,0.088401996] .
$$

Calculation 17.4.6.4. For quarters $Q$, the second partials of $\operatorname{sol}(Q)$ lie in

$$
\text { [-0.1040074557, 0.1384785805]. }
$$

Calculation 17.4.6.5. For quasi-regular tetrahedrons $T$, the second partials of gma $(T)$ volume lie in

$$
\text { [-0.0968945273, 0.0512553817]. }
$$

Calculation 17.4.6.6. For quarters $Q$, the second partials of $g$ ma $(Q)$ volume lie in

$$
\text { [-0.1362100221, 0.1016538923]. }
$$

Calculation 17.4.6.7. For quasi-regular tetrahedrons $T$, the second partials of $\operatorname{vor}(T)$ volume lie in

$$
[-0.1856683356,0.1350478467] \text {. }
$$

Calculation 17.4.6.8. For quarters $Q$, the second partials of $\operatorname{vor}(Q)$ volume lie in

$$
[-0.2373892383,0.1994181009] \text {. }
$$


The computed $g m a(\cdot)$ second partials then lie in

$$
[-0.2119591984,0.2828323141]
$$

for quasi-regular tetrahedrons and quarters.

Likewise, the computed vor( $\cdot)$ second partials then lie in

$$
\text { [-0.7137209962, 0.8691765157], }
$$

for quasi-regular tetrahedrons and quarters.

\section{Acknowledgments}

This paper is a revised version of the author's Ph.D. thesis [Fer] at the University of Michigan. The author thanks Tom Hales, Jeff Lagarias, and the referees for their many contributions to this revision.

\section{References}

[Fer] S. P. Ferguson, Sphere Packings, V, Thesis, University of Michigan, 1997.

[Ha6] T. C. Hales, Sphere packings, I, Discrete Comput. Geom. 17 (1997), 1-51.

[HF] T. C. Hales and S. P. Ferguson, A formulation of the Kepler conjecture, Discrete Comput. Geom., this issue, pp. 21-70

[IEEE] IEEE Standard for Binary Floating-Point Arithmetic, ANSI/IEEE Std. 754-1985, IEEE, New York.

Received November 11, 1998, and in revised form September 12, 2003, and July 25, 2005.

Online publication February 27, 2006. 